\title{
PRODUÇÃO CIENTÍFICA NACIONAL SOBRE O ENSINO DE LUTAS NO AMBIENTE ESCOLAR: ESTADO DA ARTE
}

\author{
Guilherme da Silva Gasparotto, Universidade Federal do Paraná - UFPR, Paraná - \\ Brasil \\ Sérgio Luiz Carlos dos Santos, Universidade Federal do Paraná - UFPR, Paraná - Brasil
}

\begin{abstract}
RESUMO
Objetivou-se verificar quantitativamente a produção científica nacional sobre o ensino de lutas na escola. Foi realizada busca sistematizada em bases indexadoras de periódicos científicos nacionais, bem como em todas as revistas de estrato A e B do sistema Qualis/Capes. A busca retornou 11 artigos que foram categorizados em: ensino de lutas na formação do professor; possibilidade de abordagens das lutas na escola; benefícios proporcionados pela vivência das lutas na escola. Foi possível perceber que o número de pesquisas relacionadas ao ensino de lutas nos últimos dez anos é pequeno e há necessidade de aumento quantitativo de pesquisas capazes de desenvolver o tema na escola visando o encorajamento dos professores de Educação Física na utilização desta ferramenta pedagógica.
\end{abstract}

Palavras-Chave: Ensino; Revisão; Artes marciais.

\section{NATIONAL SCIENTIFIC PRODUCTION ON TEACHING OF FIGHTS IN SCHOOL ENVIRONMENT: STATE OF THE ART}

\begin{abstract}
The objective was to verify quantitatively the national scientific production on teaching fights at school. Was performed systematic search in indexing databases of national scientific journals and in all journals strata A and B in system Qualis/Capes. The search returned 11 articles that were categorized to: teaching fights in teacher undergraduation; possibility of address the issue fights at school; benefits provided by the experience of fights learning at school. It could be observed that the number of researches related to teaching fight the past ten years is little and there need to increase quantitative research can develop the issue in school to the encouragement of Physical Education teachers in using this educational tool.
\end{abstract}

Key-Words: Teaching; Review; Martial arts. 


\section{PRODUCCIÓN CIENTÍFICA NACIONAL DE ENSEÑANZA DE LUCHAS EN LA ESCOLA: ESTADO DEL ARTE}

\section{RESUMEN}

El objetivo fue determinar cuantitativamente la producción científica nacional en la enseñanza de luchas en la escuela. Búsqueda sistemática fue realizada en bases de datos de indexación de revistas científicas nacionales y en todo sistema Qualis / Capes em revistas con estratos A y B. La búsqueda ha dado 12 artículos se clasificaron en: enseñanza de luchas en la formación del profesorado; posibilidad de enfoque de luchas en la escuela, los beneficios proporcionados por la experiencia de las luchas en la escuela. Se pudo observar que el número de investigaciones relacionadas con la enseñanza de las luchas de los últimos diez años es poca y hay necesidad de aumentar la investigación cuantitativa capaz de desarrollar el tema en la escuela, al estímulo de los profesores de Educación Física en el uso de esta herramienta educativa.

Palabras-Clave: Educación; Revista; Artes marciales. 


\section{INTRODUÇÃO}

Frequentemente é apontado pela literatura que um adequado desenvolvimento de crianças e adolescentes em idade escolar, nos domínios social, psicológico, cognitivo e biológico está relacionado a efetivos estímulos externos, capazes de auxiliar nesta tarefa. ${ }^{1-2}$ Neste sentido, o ambiente escolar é apontado como espaço determinante no que diz respeito à demanda e direcionamento destes estímulos. ${ }^{3}$

Dentre as atividades oferecidas, a disciplina de Educação Física demonstra ser importante ferramenta no processo de desenvolvimento dos alunos em idade escolar, visto a quantidade e variedade de estímulos capazes de ser vivenciados neste ambiente. Segundo Rosário e Darido, ${ }^{4}$ o objeto de intervenção da Educação Física Escolar é o corpo em movimento e nesta perspectiva, o aluno tem a possibilidade de aprender sobre o mundo em que está inserido a partir de vivências proporcionadas contextualizadamente durante estas aulas.

A partir da concepção da importância de conteúdos adequados e resgate histórico-social de movimentos pertencentes ao enfoque da Educação Física, o Ministério da Educação, por meio dos Parâmetros Curriculares Nacionais, ${ }^{5}$ baseados em estudos de diversos autores, ${ }^{6-8}$ define como conteúdos estruturantes da disciplina: a ginástica, os jogos, o esportes, a dança e a luta sendo que tais assuntos devem ser discutidos, ensinados e apreendidos de maneira a relacioná-los aos diversos conteúdos articuladores.

Apesar de se entender que a abordagem dos diferentes conteúdos é igualmente importante para disponibilizar amplo acervo de movimentos e bases culturais apresentadas por cada tema, por vezes algumas modalidades, em geral "esportes coletivos”, são supervalorizadas frente às outras no processo de ensino-aprendizagem. ${ }^{9}$ Tal fato pode ser reflexo ou refletir pouco empenho e entusiasmo de estudiosos e pesquisadores na produção de estudos referentes aos conteúdos "desvalorizados". 
Neste contexto, é possível citar o caso do ensino do conteúdo lutas na escola, em que a insegurança causada pela valorização do conhecimento técnico frente aos outros domínios da cultura corporal acerca do tema poderia afastar os professores desta abordagem e, consequentemente, no interesse à pesquisa sobre o assunto.

Com base nestes pressupostos, torna-se importante verificar quanto e como é representado cientificamente o estudo destas modalidades, subestimadas no âmbito prático do ensino escolar, com intuito de entender quais os fatores capazes de influenciar a abordagem e a qualidade desta pelo professor de Educação Física escolar. Nesta perspectiva o objetivo deste estudo foi verificar quantitativamente a produção científica nacional sobre a temática do ensino de lutas na escola e os principais temas desenvolvidos por estes estudos.

\section{MATERIAIS E MÉTODOS}

Esta pesquisa trata-se de uma revisão sistemática de estudos publicados em periódicos nacionais, sobre a temática de ensino de lutas. Foram realizadas buscas nas bases de documentos científicos: Scielo, Periódicos Capes, Google Scholar no intervalo de 10 anos, período de 2004 a 2013. Na base Scielo utilizou-se os termos booleanos para os definição de "Ensino" OR “Aprendizagem" AND "Artes Marciais" OR "Lutas" OR Jogos de Oposição" OR "Jogos de Combate" AND "Escola" OR "Escolar". Tanto na base Periódicos Capes e Google Scholar foram realizadas todas as combinações possíveis entre os termos para retorno de estudos que abordassem a temática no campo de busca "Assunto".

Além das buscas nas bases de artigos científicos, foi realizada verificação minuciosa pelos periódicos relacionados no sistema Qualis/Capes. Todas as revistas com temática relacionadas à Educação Física escolar classificadas nos estratos A e B foram pesquisadas com os termos relacionados ao ensino de lutas, já mencionados.

Foram incluídos artigos que abordaram o ensino de lutas no ambiente escolar ou a discussão do tema no currículo acadêmico, que tenham sido publicados em periódicos nacionais. Os artigos selecionados para revisão foram categorizados em três eixos de 
abordagem, segundo o tema proposto: ensino de lutas na formação do professor; possibilidade de abordagens das lutas na escola e benefícios proporcionados pela vivência das lutas na escola.

A sequência metodológica para seleção dos artigos pertinentes aos critérios atribuídos foi: busca pelos termos, seleção após leitura dos títulos, seleção após leitura dos resumos e por fim seleção após leitura do artigo completo.

\section{RESULTADOS}

Além da busca pelos termos nas bases de artigos científicos foram verificadas 28 revistas com temática relacionada à Educação Física escolar.

O número de artigos selecionados em cada etapa do processo está exposto na Figura 1 abaixo.

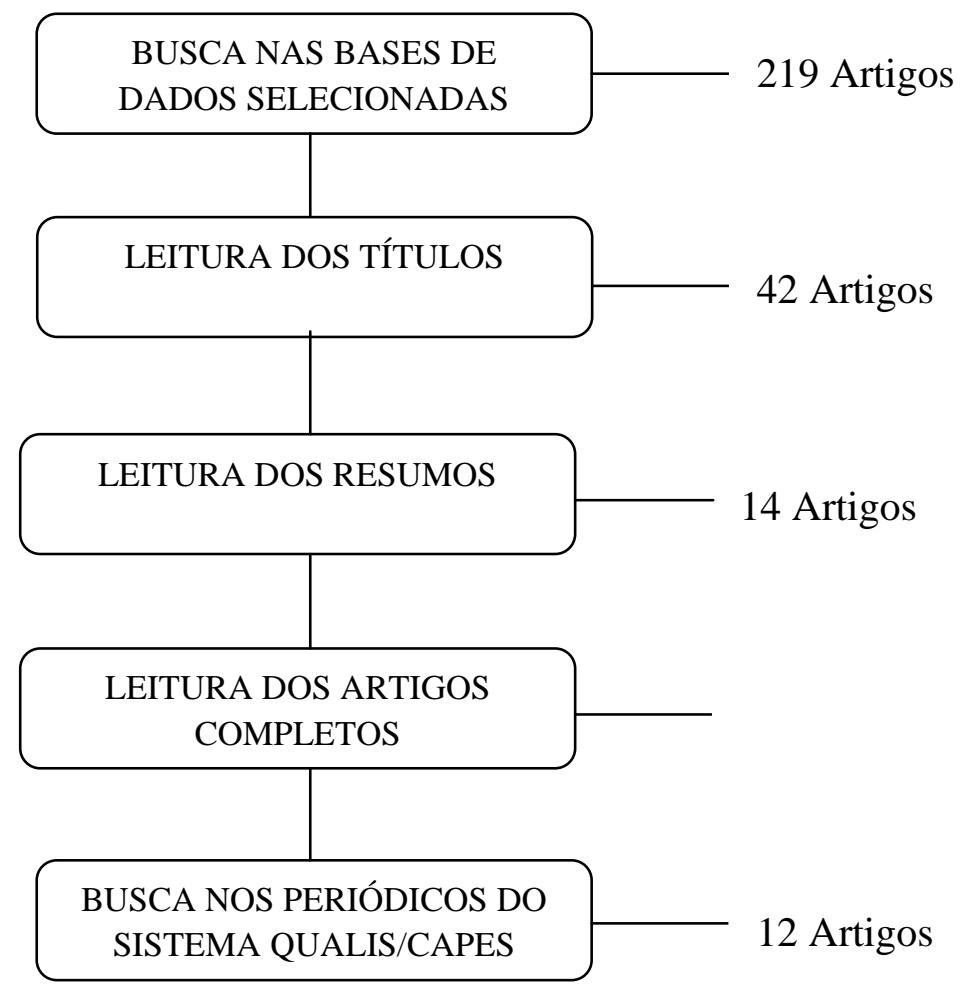

Figura 1: sistema de busca dos artigos pertinentes.

Conexões: revista da Faculdade de Educação Física da UNICAMP, Campinas, v. 11, n. 4, p. 112-124, out./dez. 2013. ISSN: 1983-9030 
As publicações relacionadas ao tema se concentraram em sete periódicos, no período de dez anos, como está apresentado na Tabela 1.

Tabela1 - Distribuição dos artigos de acordo com o periódico e ano de publicação

\begin{tabular}{|c|c|c|c|c|c|c|c|c|c|c|}
\hline PERIÓDICO & 2004 & 2005 & 2006 & 2007 & 2008 & 2009 & 2010 & 2011 & 2012 & 2013 \\
\hline Movimento & & & & 2 & & & 1 & & & \\
\hline Pensar a Prática & & & & & & & & 1 & & \\
\hline Conexões & & & & & & & & & & 1 \\
\hline Fiep Bulletin & & & & & & & & 1 & & 1 \\
\hline Motrivivência & 1 & & & & 1 & & & & & \\
\hline Mov. e Percepção & & & & & & & 1 & & & \\
\hline Cad. Form. RBCE & & & & & & & & 1 & & \\
\hline RBEFE & & & & & & & & & 1 & \\
\hline
\end{tabular}

Legenda: RBEFE: Revista Brasileira de Educação Física e Esporte.

Cad. Form. RBCE. Cadernos de Formação da Revista Brasileira de Ciências do Esporte

Os trabalhos abordaram três diferentes temáticas referentes aos domínios do ensino de lutas na escola. Os temas abordados encontram-se relacionados no Quadro 1.

Quadro 1 - Distribuição dos estudos de acordo com a temática

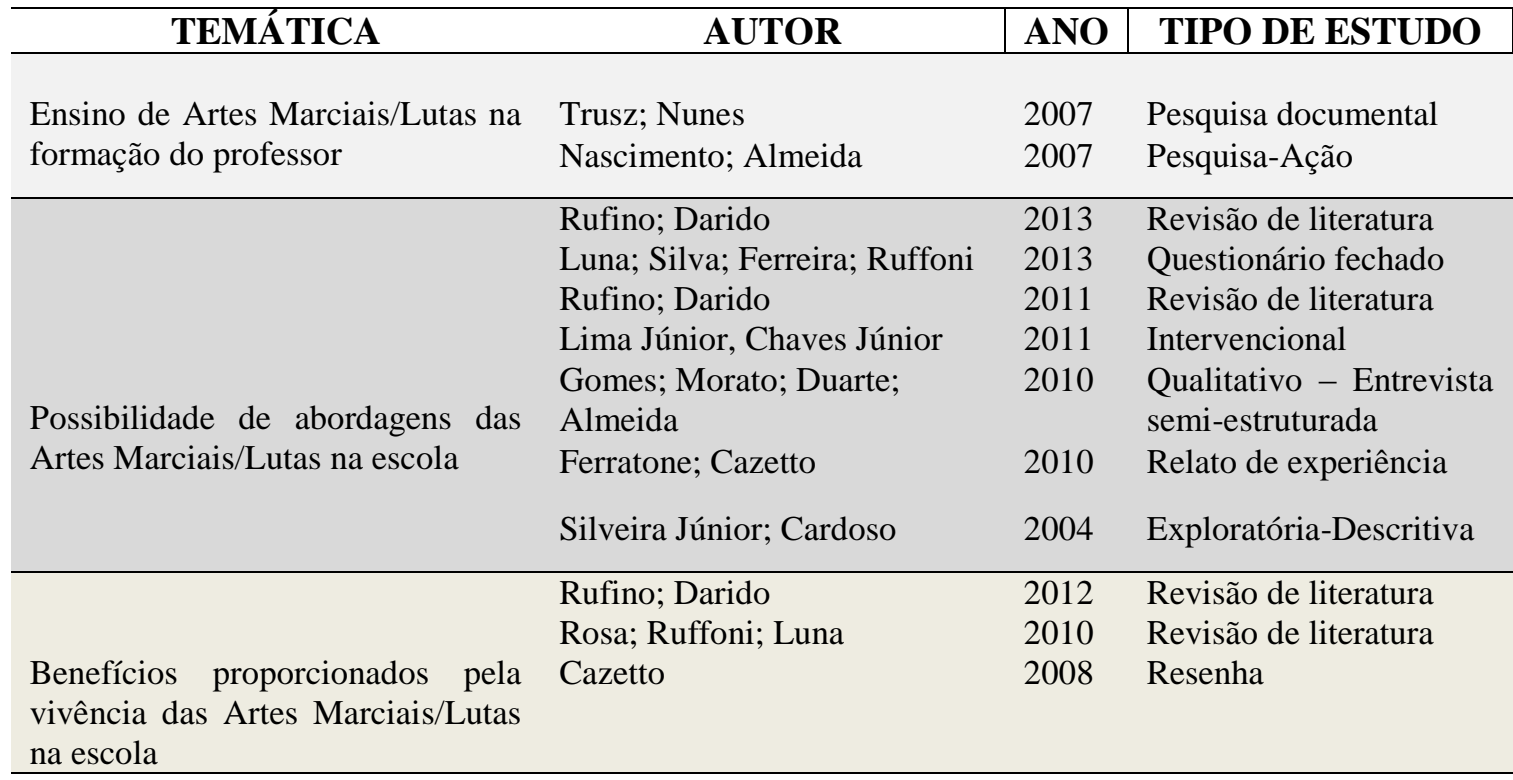

Conexões: revista da Faculdade de Educação Física da UNICAMP, Campinas, v. 11, n. 4, p. 112-124, out./dez. 2013. ISSN: 1983-9030 


\section{DISCUSSÃO}

Diante da quantidade de artigos encontrados após a busca, é possível perceber o pequeno número de publicações científicas, nos últimos dez anos, com o objetivo de investigação sobre o ensino de lutas no ambiente escolar. Diante da importância mencionada pelos PCNs,${ }^{5}$ que apontam as "lutas", como um dos assuntos fundamentais para desenvolvimento do ensino da Educação Física escolar, este dado torna-se preocupante se considerado que a base de apoio para a prática do ensino é por vezes obtida nos estudos que sugerem métodos e considerações importantes sobre o tema. Nesta perspectiva, diante de uma quantidade pequena de material à disposição do professor para consulta e julgamento da pertinência em seu contexto, a ação muitas vezes bem intencionada, é baseada no empirismo e possivelmente realizada de forma equivocada ou simplesmente não é tomada, prejudicando o público de maior interesse, o aluno, que deixa de vivenciar um conteúdo importante da cultura corporal.

Os estudos incluídos na pesquisa foram categorizados sobre três perspectivas de abordagem do ensino de lutas na escola: quanto à formação do professor que atuará no ensino, quanto às possibilidades de abordagem da temática e sobre os benefícios proporcionados pela vivência do conteúdo. Sobre a formação do professor, dois estudos abordaram criticamente a forma como é vista a disciplina de "lutas" na graduação, entre eles Trusz e Nunes ${ }^{10}$ citam a necessidade de que o curso de graduação em Educação Física proporcione ao estudante, visando disponibilizar um profissional capacitado, formação abrangente, eclética fundamentada em princípios pedagógicos, sociológicos, biológicos e filosóficos, capaz de entender as relações entre seu desenvolvimento morfo-funcional e o ser social e politizado. Neste contexto, Nascimento e Almeira ${ }^{11}$ mostram a necessidade de se entender que o professor não precisa ser um especialista ou praticante de artes marciais para desenvolver o tema de forma que os alunos tirem proveito para sua aprendizagem.

Del Vecchio e Franchini ${ }^{22}$ apontam para o fato que, a dificuldade do professor em trabalhar com o ensino de lutas se deve pela deficiência dos cursos de graduação na abordagem do conteúdo "lutas" que muitas vezes se resume em uma só modalidade, como Capoeira ou Conexões: revista da Faculdade de Educação Física da UNICAMP, Campinas, v. 11, n. 4, p. 112-124, out./dez. 2013. ISSN: 1983-9030 
Judô. Segundo o mesmo autor, em muitos casos, essas disciplinas são ministradas de forma extracurricular por ex-praticantes da modalidade e estão fora do projeto político pedagógico da instituição.

Sobre as possibilidades de abordagens no ensino de lutas na escola autores de sete estudos teceram suas considerações. Rufino e Darido ${ }^{13}$ entendem que diante das especificidades histórico-culturais, diferentemente de como muitas vezes é tratado hoje, o conteúdo "lutas" deveria ser apresentado desvinculado ao ensino do esporte na escola. Cazeto ${ }^{21}$ sinaliza ainda, o importante conteúdo articulador relacionado ao ensino desta temática, a questão na violência na escola. Não obstante, um importante foco de pesquisas e atuações práticas no ensino de artes marciais/lutas na escola que se forma hoje é a questão das mídias atuando sobre grandes eventos de Mixed Martial Arts (MMA).

A respeito do desenvolvimento das modalidades no ambiente escolar Rufino e Darido ${ }^{12}$ propõem:

É preciso que as lutas, juntamente com os outros conteúdos da Cultura Corporal sejam aplicadas em aulas de Educação Física na escola nas diversas séries, em diversos semestres, promovendo para os alunos, algo que eles ainda não têm em relação à essa temática, que é a vivência de diferentes modalidades, a apropriação crítica da cultura envolta nessas práticas e a ampliação dos conteúdos, promovidos pelas dimensões dos conteúdos.

Para os outros autores que pesquisaram as abordagens possíveis relacionadas ao ensino de lutas no ambiente escolar, parece consenso que o ensino deve ocorrer sob a perspectiva não somente do ensino das técnicas da modalidade específica como também, todo acervo cultural, sócio-afetivo e cognitivo que o tema pode proporcionar. Estes autores citam ainda a possibilidade de atividades lúdicas voltadas para os jogos de oposição ou jogos de combate, além das diversas modalidades. ${ }^{13-18}$ Santos et al. ${ }^{23}$ aponta os Jogos de Oposição como uma metodologia recente capaz de promover o ensino dos esportes de combate, desvinculando do estereótipo de violência e competitividade, concebido previamente. Segundo este autor, a terminologia artes marciais deve ser revista quando proposto no ensino escolar, visto sua origem na mitologia - Marte: Deus da Guerra. Da mesma forma a Conexões: revista da Faculdade de Educação Física da UNICAMP, Campinas, v. 11, n. 4, p. 112-124, out./dez. 2013. ISSN: 1983-9030 
terminologia luta, já que luta é um termo genérico e pode apresentar diversas conotações, a sugestão seria de denominar este conteúdo como jogos de oposição.

Por fim, três estudos propuseram os benefícios proporcionados pela aprendizagem das "lutas" na escola. ${ }^{19-2}$ Sem grandes surpresas os três estudos citam a importância da prática das Artes Marciais/Lutas no contexto escolar quanto ao desenvolvimento motor e cognitivo, assim como é sabido da importância das atividades físicas para o desenvolvimento destas variáveis, quando administradas adequadamente. Entretanto, de forma especial, o conteúdo é abordado pelos autores quanto à possibilidade de trabalhar fatores inerentes à prática das "Lutas". Como aponta Rosa et al. ${ }^{20}$, situações de medo, autocontrole emocional, ansiedade, canalização de agressividade, respeito e hierarquia são especialmente vivenciados neste tipo de atividade. Além disto, estes autores lembram ainda, a imensa carga cultural e filosófica que as Artes Marciais trazem de contribuição dos outros povos para o Brasil, a se citar a Capoeira, o Judô, o Jiu-Jítsu, entre tantas outras. Rufino e Darido ${ }^{19}$ contribuem para a prática do professor quando expõem que o ensino deste conteúdo deve estar pautado em eixos norteadores: por que ensiná-las? o que ensinar das Lutas? como ensiná-las? e como avaliá-las? Com base nisto se poderá efetuar o julgamento crítico necessário para um ensino efetivo.

\section{CONCLUSÕES}

Diante deste estudo, foi possível perceber que o número de pesquisas relacionadas ao ensino de lutas nos últimos dez anos é pequeno perto da importância dada à temática pelas próprias instituições que regulamentam e sugerem os temas inerentes ao estudo da Educação Física escolar no Brasil. Além disso, observou-se que elas estão concentradas em somente alguns periódicos nacionais.

A maior quantidade destes estudos discutem as possibilidades de abordagens com o ensino do conteúdo apontando para o direcionamento de menor valorização das técnicas específicas das modalidades e possibilidade de desenvolvimento de valores socioculturais, afetivos e cognitivos. Não obstante, possibilita o desenvolvimento de assuntos evidentes na Conexões: revista da Faculdade de Educação Física da UNICAMP, Campinas, v. 11, n. 4, p. 112-124, out./dez. 2013. ISSN: $1983-9030$ 
sociedade contemporânea, como atuação midiática em grandes eventos e a violência gratuita.

Parte dos estudos tratou da contribuição pedagógica do ensino do tema, como o desenvolvimento psicomotor perante a prática, além da carga cultural advinda das artes marciais praticadas no Brasil.

Pensando na formação adequada dos profissionais, dois autores abordaram a metodologia de trabalho na disciplina de lutas durante a graduação do profissional, atribuindo a pouca e/ou ineficiente prática na escola à deficiência de desenvolvimento desta disciplina na durante a formação.

Este estudo sugere a necessidade de aumento quantitativo de pesquisas capazes de desenvolver o tema lutas nas escolas, sob a perspectiva de uma sistematização, visando o encorajamento dos professores de Educação Física na utilização desta ferramenta pedagógica ainda pouco explorada diante da falta de conhecimento e insegurança dos profissionais.

\section{REFERÊNCIAS}

${ }^{1}$ SCALHA, T. B. et. al. A importância do brincar no desenvolvimento psicomotor: relato de experiência. Revista de Psicologia da UNESP, Assis, v. 9, n. 2, p. 79-92, 2010.

${ }^{2}$ KREBS, J. R.; DESIREE, C. J.; ZÊNITE, M. Contexto de desenvolvimento e a percepção espacial de crianças. Movimento, Porto Alegre, v. 17, n. 1, p. 195-212, 2011.

${ }^{3}$ DAOLIO, J. Educação Física e o conceito de cultura. Campinas: Autores Associados, 2004. 
${ }^{4}$ ROSÁRIO, L. F. R.; DARIDO, S. C. A sistematização dos conteúdos da Educação Física na escola: a perspectiva dos professores experientes. Motriz, Rio Claro, v. 11 n. 3 p.167$178,2005$.

${ }^{5}$ BRASIL. Ministério da Educação e Desporto. Parâmetros Curriculares Nacionais: Educação Física. Brasília, 1998.

${ }^{6}$ BETTI, M. Janela de vidro: educação física e esportes. 1997. Tese (Doutorado em Educação Física) - Universidade Estadual de Campinas, Campinas, 1997.

${ }^{7}$ BRACHT, V. Educação Física e aprendizagem social. Porto Alegre: Magister, 1992.

${ }^{8}$ CASTELLANI FILHO, L. Considerações acerca do conhecimento (re)conhecido pela Educação Física escolar. Revista Paulista de Educação Física, São Paulo, supl. n. 1, 1995.

${ }^{9}$ SANTOS, M. A. G. N.; NISTA-PICCOLO, V. L. O esporte e o ensino médio: A visão dos professores de educação física da rede pública. Revista Brasileira de Educação Física e Esporte, São Paulo, v. 25, n. 1, p. 65-78, 2011.

${ }^{10}$ TRUSZ, R. A.; NUNES, A. V. A evolução dos esportes de combate no currículo do curso de Educação Física da UFRGS. Movimento, Porto Alegre, v. 13, n. 1, p. 179-204, 2004.

${ }^{11}$ NASCIMENTO, P.; ALMEIDA, L. A. tematização das lutas na Educação Física Escolar: restrições e possibilidades. Movimento, Porto Alegre, v. 13, n. 3, p. 91-110, 2007.

${ }^{12}$ RUFINO, L. G. B.; DARIDO, S. C. Possíveis diálogos entre a educação física escolar e o conteúdo das lutas na perspectiva da cultura corporal. Conexões, Campinas, v. 11, n. 1, p. 145-170, 2013.

Conexões: revista da Faculdade de Educação Física da UNICAMP, Campinas, v. 11, n. 4, p. 112-124, out./dez. 2013. ISSN: 1983-9030 
${ }^{13}$ RUFINO, L. G. B.; DARIDO, S. C. A separação dos conteúdos das "lutas" dos "esportes" na Educação Física escolar: necessidade ou tradição? Pensar a Prática, Goiânia, v. 14, n. 3, p 1-17, 2011.

${ }^{14}$ LUNA, I. M. et. al. Lutas na escola: uma visão dos acadêmicos em educação física. Fiep Bulletin. Disponível em:

< http://www.fiepbulletin.net/index.php/fiepbulletin/article/view/2905>. Foz do Iguaçu, v. 83, 2013.

${ }^{15}$ LIMA JUNIOR, H. C.; CHAVES JUNIOR, S. R. Possibilidades das lutas como conteúdo na educação física escolar: o confrontamento em uma abordagem pedagógica com alunos de $6^{\text {a }}$ série em um colégio estadual do município de Guarapuava-PR. Cadernos de Formação RBCE, Florianópolis, v. 2, n. 1, p. 69-80, 2011.

${ }^{16}$ GOMES, M. S. P. et. al. Ensino das lutas: dos princípios condicionais aos grupos situacionais. Movimento, Porto Alegre, v. 16, n. 02, p. 207-227, 2010.

${ }^{17}$ FERRATONE, S.; CAZETTO, F. F. Lutas e Artes Marciais na Escola: um relato de experiência sobre o aprofundamento nos conteúdos. Movimento e Percepção, Espírito Santo do Pinhal, v. 11, n.17, 2010.

${ }^{18}$ SILVEIRA JÚNIOR, E. R.; CARDOSO C. L. QIGONG: Uma contribuição para a Educação Física escolar. Motrivivência, Florianópolis, n. 23, p. 83-101, 2004.

${ }^{19}$ RUFINO, L. G. B.; DARIDO, S. C. Pedagogia do esporte e das lutas: em busca de aproximações. Revista Brasileira de Educação Física e Esporte, São Paulo, v. 26, n. 2, p. 283-300, 2012.

${ }^{20}$ ROSA, T. S.; RUFFONI, R.; LUNA, I. Lutas na escola: valiosa ferramenta pedagógica para o segundo segmento do ensino fundamental. Fiep Bulletin. Disponível em: 
<http://www.fiepbulletin.net/index.php/fiepbulletin/article/view/236>. Foz do Iguaçu, v. 81, 2011.

${ }^{21}$ CAZETTO, F. F. Lutas e Artes Marciais na Escola. Motrivivência, Florianópolis, n. 31, p. 251-255, 2008.

${ }^{22}$ DEL VECCHIO, F. B.; FRANCHINI, E. Lutas, artes marciais e esportes de combate: Possibilidades, Experiências e Abordagens no currículo da Educação Física. In: Souza NETO, S.; HUNGER, D. Formação profissional em Educação Física: estudos e pesquisas, Rio Claro, Biblioética, 2006.

${ }^{23}$ SANTOS, S. L. C. et. al. Juegos de oposición: nuevas metodologias para la enseñanza de deportes de combate . Revista de Ciencias del Deporte, v. 7 (Suppl.), p. 45-62, 2011.

${ }^{24}$ MACHADO, S. T. et. al. As práticas de desinvestimento pedagógico na Educação Física escolar. Movimento, Porto Alegre, v. 16, n. 2, p. 129-147, 2010.

Recebido em: 12 jul. 2013

Aceito em: 19 nov. 2013 Contato: Guilherme da Silva Gasparotto guilhermegptt@gmail.com 